\title{
Distributed dynamic strain sensing via perfect periodic coherent codes and a polarization diversity receiver
}

\author{
Juan José Mompó, Lihi Shiloh, Nadav Arbel, Nadav Levanon, Life Fellow, IEEE \\ Alayn Loayssa, Senior Member, IEEE, Avishay Eyal, Senior Member, IEEE,
}

\begin{abstract}
Rayleigh scattering-based dynamic strain sensing with high spatial resolution, fast update rate and high sensitivity is highly desired for applications such as structural health monitoring and shape sensing. A key issue in dynamic strain sensing is the trade-off between spatial resolution and the Signal to Noise Ratio (SNR). This trade-off can be greatly relaxed with the use of coding. A sequence of optical pulses is injected into the fiber and the detected backscattered signal is cross-correlated with the original signal. With the use of coding, SNR is indeed improved, but if the sequence is not well chosen, the resulting peak to sidelobe ratio (PSR) can be rather low. An excellent choice of codes are biphase Legendre sequences which offer near perfect periodic autocorrelation (PPA). Other common issues in Rayleigh scattering-based sensing techniques are signal fading and dynamic range. The former issue can occur due to destructive interference between lightwaves that are scattered from the same spatial resolution cell and, in coherent detection schemes, when the polarization states of the backscattered light and the reference light are mismatched. The latter issue is a concern in phase sensitive schemes which require signal jumps not to exceed $2 \pi$. In this paper, a biphase Legendre sequence with 6211 pulses is used in conjunction with polarization diversity scheme and a PM fiber. The setup provides two independent measurements of the sensing fiber complex profile and achieves highly sensitive, distributed dynamic strain sensing with very low probability of fading. In addition, the system can handle both very large perturbation signals and very small perturbation signals. The system operated at a scan rate of $\sim 107 \mathrm{kHz}$ and achieved spatial resolution of $\sim 10 \mathrm{~cm}$ and sensitivity of $\sim 1.1 \mathrm{mrad} / \sqrt{\mathrm{Hz}}$. The ratio between the powers of the maximum and minimum excitations that can be measured by the system is $136 \mathrm{~dB}$.
\end{abstract}

Index Terms-Fiber optical sensors, Optical time domain reflectometry, Phase modulation, Rayleigh scattering

\section{INTRODUCTION}

In recent years, Distributed Optical Fiber Sensors (DOFS) have been intensively studied because of their advantages over

Juan José Mompó is with the Department of Electrical and Electronic Engineering and the Institute of Smart Cities, Universidad Pública de Navarra, Pamplona 31006, Spain, (juanjose.mompo@unavarra.es).

Lihi Shiloh is with the Electrical Engineering Faculty, Tel-Aviv University, Tel Aviv 6997801, Israel (lihishiloh@mail.tau.ac.il).

Nadav Arbel is with the Electrical Engineering Faculty, Tel-Aviv University, Tel Aviv 6997801, Israel (nadavarbel@tauex.tau.ac.il).

Nadav Levanon is with the Electrical Engineering Faculty, Tel-Aviv University, Tel Aviv 6997801, Israel (nadav@eng.tau.ac.il).

Alayn Loayssa is with the Department of Electrical and Electronic Engineering and the Institute of Smart Cities, Universidad Pública de Navarra, Pamplona 31006, Spain, (alayn.loayssa@unavarra.es).

Avishay Eyal is with the Electrical Engineering Faculty, Tel-Aviv University, Tel Aviv 6997801, Israel (avishay@eng.tau.ac.il).

Manuscript received April 19, 2005; revised August 26, 2015. traditional point sensors. One of the techniques which has been drawing significant interest, in particular for applications of Distributed Acoustic Sensing (DAS), is Rayleighbased phase-sensitive Optical Time-Domain Reflectometry $(\Phi-O T D R)$ since it is able to provide fast measurement of dynamic strain with high sensitivity. A major limitation of this technique is the trade-off between its spatial resolution and Signal-to-Noise Ratio (SNR). This is because the backscattered power is proportional to the temporal width of the pulse, but the spatial resolution degrades when the pulse width increases [1]. An alternative technique that circumvents this trade-off is Optical Frequency Domain Reflectometry (OFDR) [2]. This technique offers high SNR along with high spatial resolution, but its implementation is challenging due to the need to generate highly accurate linearly frequency-modulated interrogation waveform during each scan. Another technique that alleviates the above-mentioned trade-off is Optical Pulse Compression Reflectometry (OPCR). In this technique a pulse with Linear Frequency Modulation (LFM) is launched into the fiber and the backscattered signal is analyzed with a matched-filter [3]. The main challenge which OPCR methods need to overcome is the undesired generation of sidelobes by the matched-filtering operation. Some techniques, such as pulse apodization [4], can improve the Peak to Sidelobe Ratio (PSR) but the suppression of the sidelobes generally comes at the expense of spatial resolution. Finally, another family of techniques which can achieve good SNR alongside with high spatial resolution is based on radar-type coding. An early version of this approach was studied by Nazarthy et. al. [5] which used complementary correlation pairs, also known as Golay codes, in order to improve the performance of an incoherent OTDR system for measurement of the static backscatter profile of an optical fiber.

The implementation of coding techniques in DOFS is not limited to Rayleigh-based optical reflectomery. It has been demonstrated to be a powerful tool in distributed Brillouin scattering sensors as well [6], [7], [8], [9]. In the realm of DAS, coding has been studied in recent years by several authors[10], [11]. A critical consideration in the choice of a coding technique is the PSR it achieves. A family of codes that offers optimal PSR are the Perfect Periodic Autocorrelation (PPA) codes. In theory, these codes have no side-lobes at all, namely, their inherent PSR is infinite. Moreover, as suggested by their 
name, the processing of the data, which is acquired via a PPA code, involves periodic cross-correlation of the captured data. In contrast with non-periodic codes, this can be implemented directly using the highly efficient FFT and IFFT. Recently the use of a PPA code for highly sensitive dynamic strain measurement and for laser range finding was demonstrated by part of the authors of this papers [12], [13].

Another fundamental issue that affects coherent Rayleigh reflectometric systems is signal fading. Fading may result from two different origins. The first one (Rayleigh fading) is destructive interference between lightwaves that are scattered from the same spatial resolution cell. The second one (polarization fading) occurs in coherent detection schemes when the polarization states of the backscattered light and the reference light are mismatched. In both cases fading may lead to SNR degradation and even to complete loss of signal. Several methods have been proposed in order to alleviate Rayleigh fading. A common approach is to interrogate the fiber with multiple wavelengths in order to generate statistically independent reflection profiles and to combine them judiciously to achieve optimal SNR [14]. This approach is successful but requires a complex setup due to the need to generate, modulate and acquire multiple wavelengths. Polarization fading is often resolved by using polarization diversity configurations [15].

Finally, a third problem that $\Phi$-OTDR presents is related to the measurement of large signals. In systems where optical phase is used to quantify strain, the maximum phase jump, between two consecutive measurements in the same location, is limited to $2 \pi$ due to phase unwrapping ambiguity. This problem limits the maximum slew rate (phase change per unit time) that can be measured. As a consequence, direct phase analysis may not be suitable in many important applications such as Structural Health Monitoring (SHM) in aircrafts where the strain developed in the wings, between two consecutive measurements, typically leads to phase variations which greatly exceeds $2 \pi$.

This paper describes a modified version of the technique presented in [12]. It is based on PPA codes, polarization diversity interrogator and a Polarization Maintaining (PM) sensing fiber. This combination facilitated fast dynamic strain measurement with low probability for Rayleigh fading and free of polarization fading. Rayleigh fading was mitigated by judiciously combining the backscatter signals from the two principle axes of the PM fibers. For this approach to succeed the two Rayleigh backscatter signals needed to be uncorrelated. The condition for uncorrelated responses is given and the effectiveness of the approach is demonstrated. Polarization fading was avoided due to the polarization diversity receiver. The new setup, in conjunction with the use of a weighted sum of polarization responses, led to another advantage of the proposed method: increased dynamical SNR and, in turn, enhanced dynamical sensitivity. Finally, the subtraction of the polarization phase responses enabled measurement of exceedingly large phase variations which could not be measured by a single polarization (or their weighted sum).

The system operated at a scan rate of $107.34 \mathrm{KHz}$, equal to the inverse of the temporal length of the code. Due to memory limitations of our scope, however, it was set to acquire the scans at a reduced rate of $16772 \mathrm{~Hz}$, so that longer signals could be recorded. The system was tested with a sinusoidal excitation of $130 \mathrm{~Hz}$ at a distance of $144 \mathrm{~m}$ with amplitudes between $1 \mathrm{rad}_{p p}$ and $1085 \mathrm{rad}_{p p}$. The spatial resolution was $0.1 \mathrm{~m}$, the achieved sensitivity $\sim 1.1 \mathrm{mrad} / \sqrt{\mathrm{Hz}}$ and the ratio between the powers of the maximum and minimum signals that the system could measure (not simultaneously) was 136 $\mathrm{dB}$.

\section{THEORY}

The use of codes with good PSR for alleviating the tradeoff between spatial resolution and SNR has been previously studied in the context of radar signals [16]. Binary antipodal Phase Shift Keying (PSK) codes are pseudorandom sequences whose elements are taken from the basic set $\{-1,+1\}$. msequences and Legendre-sequences are two examples of such codes which attain good PSRs as their length, $N$, increases. An interesting property of these codes is that they can become PPA codes when their basic set is replaced by $\{\exp (j \Phi),+1\}$ where $\Phi$ is taken as [17]:

$$
\Phi=\cos ^{-1}\left(-\frac{N-1}{N+1}\right)
$$

In the Legendre case $N$ can be any prime number that obeys $N=4 k-1$, where $k$ is an integer. It can be seen that as $N$ increases $\Phi$ approaches $\pi$ and the code approaches its antipodal version. As antipodal codes are simpler to implement optically (in comparison with general binary PSK codes) these were the codes that were used in this work. The optical interrogation lightwave is a sequence of pulses with identical envelopes and pseudo-randomly alternating signs which can be expressed as:

$$
E_{i n}(t)=\sum_{k=1}^{N} C_{k} E_{0} a\left(t-k T_{b i t}\right)
$$

where $E_{0}$ is the amplitude of the optical field, $C_{k}$ is the code element at position $k$ and $a(t)$ describes the envelope of a single pulse. The pulse envelope, which is normalized to unit energy, attains a positive value between $\left[-T_{b i t} / 2, T_{b i t} / 2\right]$ and vanishes elsewhere. Thanks to the properties of the selected code the periodic autocorrelation of $E_{i n}(t)$ is near perfect, and given by:

$$
R(\tau) \approx E_{0}^{2} N R_{a}(\tau)
$$

where $R_{a}(\tau)$ is the autocorrelation of the elementary pulse. In DAS, the field which is backscattered from the Fiber Under Test (FUT) can be described as:

$$
E_{F U T}(t)=\int_{0}^{L} \rho\left(z^{\prime}\right) E_{i n}\left(t-\frac{2 z^{\prime}}{v_{g}}\right) d z^{\prime}
$$

where $\rho(z) \approx r(z) \exp (-j 2 \beta z)$ is the complex backscatter coefficient of the fiber. It describes both the local backscatter complex amplitude, $r(z)$, and the optical phase acquired by the light during its trip to point $z$ and back. $v_{g}$ is the group velocity of the light and $L$ is the total fiber length. The local backscatter complex amplitude is assumed to be a white 
spatial random process, namely, $<r\left(z^{\prime}\right) r^{*}\left(z^{\prime \prime}\right)>\propto \delta\left(z^{\prime}-z^{\prime \prime}\right)$. Optical detection of the reflected signal at the output of the FUT is done with an I/Q receiver [18]. Then the detected signal is sampled and cross-correlated with the transmitted signal (2) yielding:

$$
R_{F U T}(t)=A \int_{0}^{L} \rho\left(z^{\prime}\right) R_{a}\left(t-\frac{2 z^{\prime}}{v_{g}}\right) d z^{\prime}
$$

where $A$ is a constant that includes parameters such as number of pulses in the code and gain of the coherent receiver. Equation (5) can be understood as a convolution between the complex profile of the fiber and the autocorrelation of the elementary pulse. The estimated fiber profile, $R_{F U T}\left(t=2 z / v_{g}\right)$, is measured repeatedly and the DAS signal is extracted from its phase variations with position and time.

In the presence of Rayleigh and polarization fading the SNR with which the DAS signal is measured varies randomly with $z$. To overcome this the proposed setup uses a PM sensing fiber and a polarization diversity detection scheme. Hence, at each scan period the system generates two simultaneous measurements of the sensing fiber which correspond to its two principal axes. The polarization maintaining configuration of the setup prevents the occurrence of polarization fading. The probability for Rayleigh fading can also be significantly reduced provided the two measured fiber profiles are uncorrelated. It is shown in appendix A that this condition is satisfied if $\Delta \beta=\beta_{y}-\beta_{x} \gg \pi / L_{a}$ (where $\beta_{x}$ and $\beta_{y}$ denote the propagation coefficients of the fiber's principal axes) where $L_{a}$ can be approximated by the length of the elementary pulse. Substituting the experimental values we obtain $\Delta \beta=2 \pi \Delta n / \lambda \approx 4.05 \cdot 10^{3} \mathrm{rad} / \mathrm{m}$ where $\pi / L_{a} \approx 20.95$ $\mathrm{rad} / \mathrm{m}$ and hence the condition is satisfied.

To obtain a signal from the repetitive measurements of $R_{F U T}\left(2 z / v_{g}\right)$ which represents the external perturbation to the fiber it is common to estimate the spatial derivative of its phase. In our case we obtain two differential phase signals which correspond to the two measured polarizations. In the case of small perturbations the two measured signals are approximately equal as the difference between them is in the order of $\Delta \beta \Delta l$ where $\Delta l$ is the elongation of a resolution cell due to the perturbation. In order to mitigate fading, both signals are combined with inverse-variance weighting according to [19]:

$$
\Delta \phi=A_{x} \Delta \phi_{x}+A_{y} \Delta \phi_{y}
$$

where

$$
A_{x, y}=\frac{\sigma_{y, x}^{2}}{\sigma_{x}^{2}+\sigma_{y}^{2}}
$$

$\sigma_{x, y}$ denote the variance of the differential phase for each polarization and $A_{x}+A_{y}=1$.

In the case of large signals, a problem may occur if the phase jump, in a given location, between two consecutive scans is bigger than $2 \pi$. In such a case, a phase difference of $2 \pi k+\varepsilon$, where $k$ is an integer, will be measured as $\varepsilon$. Accordingly, for a sinusoidal phase signal, the maximum amplitude which can be measured without errors is given by $A=f_{s} / f$ where $f_{s}$ is the scan frequency and $f$ is the frequency of the phase signal. To enable measurement of large signals we propose to use the difference between the differential phases of the two polarization backscatter profiles:

$$
\Delta \phi_{\text {diff }}=\left(\phi_{i+\Delta i}^{x}-\phi_{i}^{x}\right)-\left(\phi_{i+\Delta i}^{y}-\phi_{i}^{y}\right)
$$

where $\phi^{x, y}$ represents the value of optical phase for horizontal and vertical polarizations, and $i$ and $i+\Delta i$ the spatial points between which the phase difference is calculated. $\Delta \phi_{\text {diff }}$ is proportional to the applied strain and is orders of magnitude smaller than $\Delta \phi$.

\section{EXPERIMENTAL SETUP}

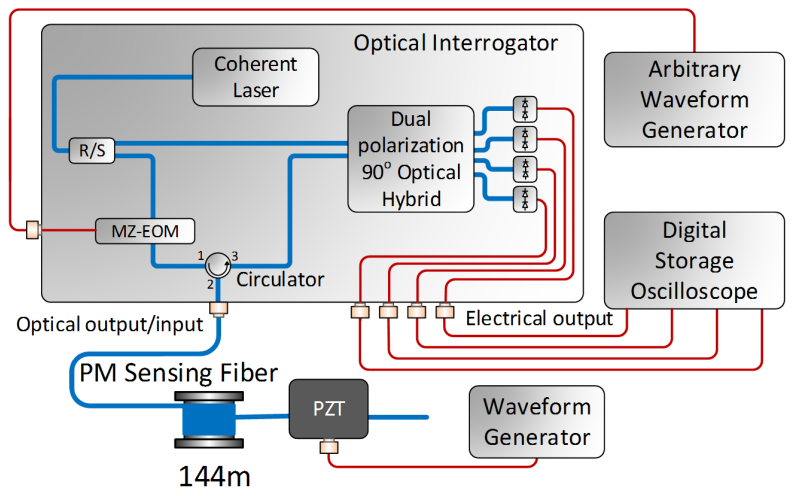

Fig. 1. The experimental setup. Blue lines indicate PM fibers and red lines indicate electrical lines.

Fig. 1 depicts schematically the optical setup used in this work. An ultra-coherent laser emitted CW light with linewidth of $\sim 0.1 \mathrm{kHz}$ and power of $21 \mathrm{dBm}$. Then, a 90:10 PM coupler $(\mathrm{R} / \mathrm{S})$ split the optical signal into two branches. One of these, which was used as a reference, was sent directly to a Dual Polarization Optical Hybrid (DPOH). The other output of the coupler was used as a sensing arm. A PM Mach-Zehnder Electro-Optic Modulator (MZ-EOM), that was biased for carrier suppression (minimum transmission) was used to generate the interrogation waveform. The MZ-EOM was driven by a binary antipodal PSK sequence of returnto-zero (RZ) pulses. The envelope of the basic pulse (bit) was raised-cosine and and its Full Width Half Maximum (FWHM) was $1.5 \mathrm{~ns}$. As usual, the MZ-EOM was driven in push-pull configuration. With these settings the light at the MZ-EOM output comprised RZ pulses which jumped between two opposite phases $(0$ and $\pi$ ). The modulated optical signal was sent to a PM circulator with linear polarization at an angle of $45^{\circ}$ with respect to the device's principal axes in order to split the power equally between the principal axes of the sensing fiber. It is assumed that cross-coupling between polarizations is negligible. The circulator diverted the signal to a 144-m PM fiber spool that had a fiber stretcher (PZT) attached at its far end. An AWG controlled the frequency and amplitude of the signal that fed the PZT. The reflected light of the optical fiber was directed by the circulator, to the DPOH. Quadrature components of the optical signal (I and Q) for both polarizations were detected using four balanced photodetectors with bandwidths of $800 \mathrm{MHz}$. The electrical signals were 
digitized and stored by an oscilloscope at a sampling rate of $2.5 \mathrm{GSamples} / \mathrm{s}$. In this work, the sequence length was chosen to be 6211 leading to a scan period of $9.3165 \mu s$.

\section{EXPERIMENTAL RESULTS}

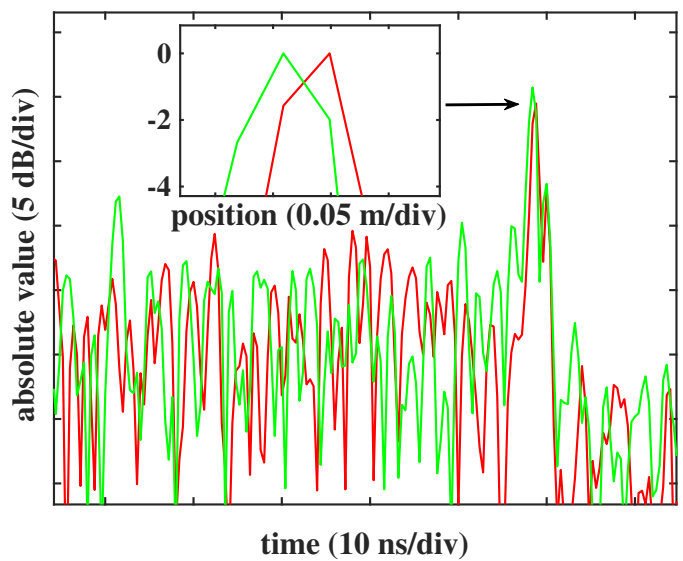

Fig. 2. Profiles of the absolute value of the reflected signal for both polarizations. The inset shows in details normalized reflections at the end of the fiber.

Fig. 2 shows the Rayleigh profiles of the two principal polarizations in the PM fiber. As mentioned above, since the two polarizations had different refractive indices they traveled different optical paths in the fiber and their Rayleigh profiles were different. This is similar to the case were the measurement is performed with two different frequencies. In the case of a PM fiber with a $\Delta n \approx 0.001$, the equivalent frequency shift is approximately $200 \mathrm{GHz}$. The peaks of the decoded signals (normalized by their maximum value), at the fiber end, can be seen in the inset of Fig. 2. The two peaks appear shifted by $5 \mathrm{~cm}$. The apparent spatial shift is due to the difference in refractive indices and mismatch in the lengths of pigtails and RF cables leading to the detectors. During post processing of the signal, this offset was compensated in order to align the spatial responses of the two polarizations. The FWHM of each of the peaks is seen to be $10 \mathrm{~cm}$. The relatively high noise level which can be seen in Fig. 2 in the section right after the fibers end reflection, is attributed to two main factors. The first factor is related to the imperfect implementation of the interrogating pulse sequence and the resulting autocorrelation sidelobes [12]. Another important factor is the relatively large bandwidth $(800 \mathrm{MHz})$ with which the backscattered signal was measured.

To demonstrate measurement of dynamic strain, $62 \mathrm{~cm}$ of the PM fiber were wrapped around a piezoelectric cylinder. The cylinder was fed with a sinusoidal signal of $130 \mathrm{~Hz}$ and with different amplitudes.

Fig. 3 shows the spectrum of the differential phase in the last $4 \mathrm{~m}$ of the sensing fiber for both polarizations and for their combination. The spatial separation used for calculating the phase difference was chosen bigger than the length of the wrapped fiber segment to ensure the linearity of the measurement [14]. Spectra were filtered using a bandpass filter

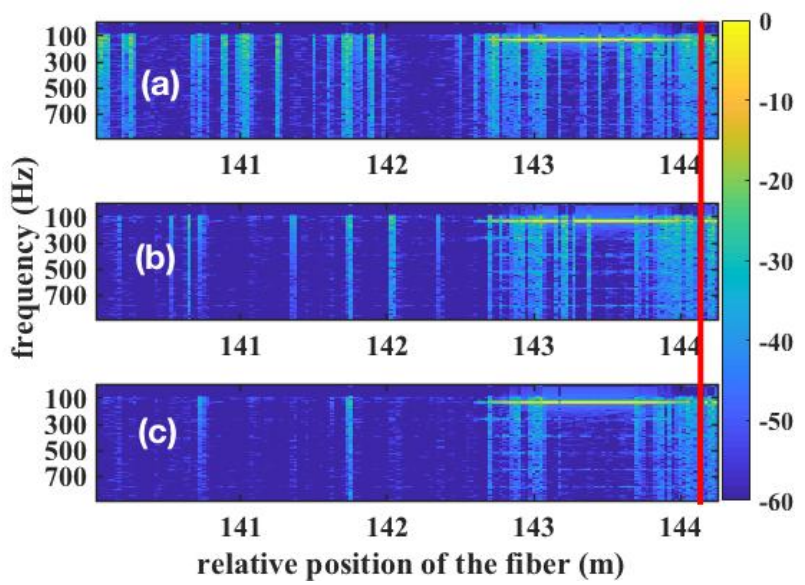

Fig. 3. Spectra of phase difference for (a) horizontal polarization, (b) vertical polarization and (c) combination of both polarizations (units in $\mathrm{dB}$ ). Red line indicates the end of the fiber.

between the frequencies of 80 and $2000 \mathrm{~Hz}$. The perturbation at $130 \mathrm{~Hz}$ can be observed at the upper right corner of each spectrum. Subplot (c) displays how signal fading induced noise was significantly reduced due to the combination of the signals in the manner described in (6) and (7).

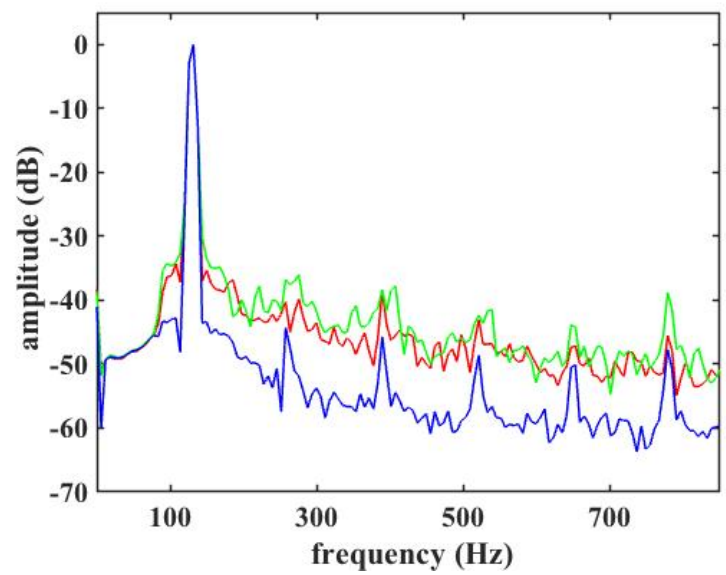

Fig. 4. Spectra of the differential phase power for horizontal polarization (red), vertical polarization (green) and their combination (blue).

Spectra of the differential phase power averaged over the location of the excitation are shown in Fig. 4. The improvement in SNR in the case of the combined response with respect to the response of each polarization alone is evident. This is primarily due to the great improvement in signal fading in the combined response case. The peak powers of these harmonics are more than $40 \mathrm{~dB}$ smaller than the peak at the fundamental frequency and the SNR of the perturbation is $\sim 50 \mathrm{~dB}$ higher than the noise floor representing sensitivity of $\sim 1.1 \mathrm{mrad} / \sqrt{\mathrm{Hz}}$. Note the presence of harmonics of the fundamental frequency of the excitation. The harmonics are attributed to mechanical resonances in the fiber stretching setup.

Fig. 5 shows examples of phase difference signals for both polarizations. The examples correspond to a location where 


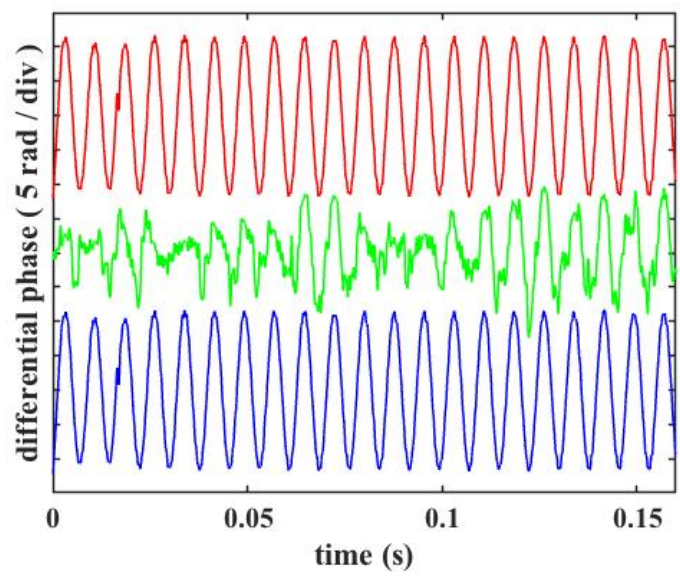

Fig. 5. Differential phase profile for horizontal polarization (red), vertical polarization (green) and their combination (blue).

one of the polarizations (vertical) had low SNR while the other polarization (horizontal) showed good SNR. Also shown is the weighted sum of the signals according to the inverse variance weighting approach ((6) and (7)). It can be seen that the combined signal retains the good SNR of the horizontal polarization and is not affected by the fading of the signal of the vertical polarization.

As previously described, when the phase difference, at a given fiber position, between two consecutive scans, exceeds $2 \pi$, unwrapping becomes ambiguous and errors occur. In order to demonstrate this limitation, the amplitude of the excitation applied to the fiber was significantly increased. Figure 6 shows the phase differences, $\Delta \phi^{x, y}$, as measured for each polarization, as a function of the excitation amplitude. Red squares and green circles indicate the phase signal measured for the vertical and horizontal polarization components respectively. Note how when the voltage applied to the PZT varied between 0 and $5 V_{p p}$ the phase signal increased linearly as expected whereas when the voltage exceeded $10 V_{p p}$ unwrapping ceased to function properly. In the same figure, blue markers refer to $\Delta \phi_{\text {diff }}$. It can be seen that the differential phase between the polarization components varies linearly with the amplitude of the excitation. The solid lines in Figure 6 are linear fits to the measured values.

\section{CONCLUSION}

In this paper, a new system for distributed dynamic strain sensing with high resolution, fast update rate and high sensitivity was presented and tested. The system used PPA code, a PM sensing fiber and a polarization diversity receiver to achieve zero polarization fading, low probability of signal fading and polarization-differential-phase mode for very large signals. The system operated at a scan rate of $\sim 107 \mathrm{kHz}$ and achieved spatial resolution of $\sim 10 \mathrm{~cm}$, sensitivity of $\sim 1.1 \mathrm{mrad} / \sqrt{\mathrm{Hz}}$ and ratio between the powers of the maximum and minimum excitations of $136 \mathrm{~dB}$.

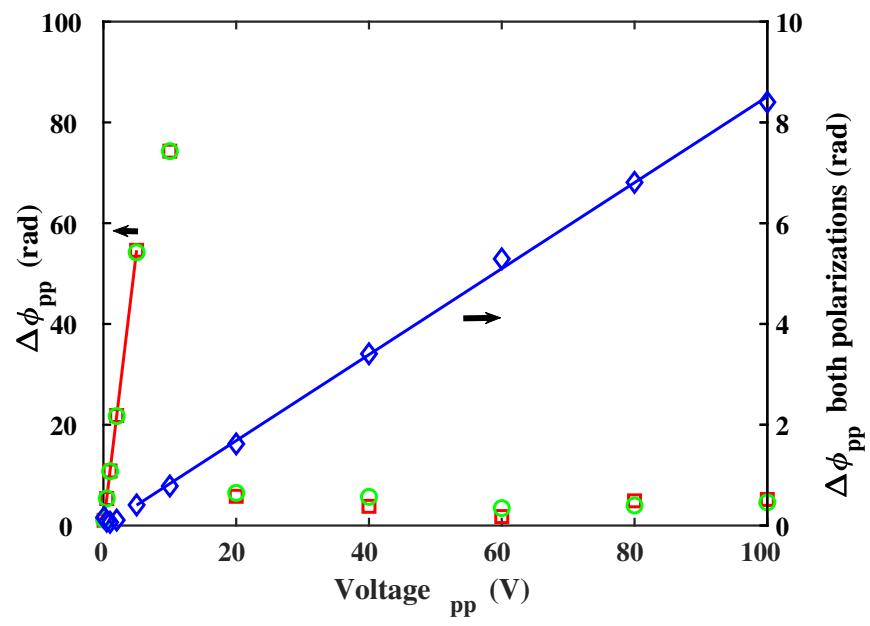

Fig. 6. Evolution of the amplitude of the detected signal for each single polarization (green and red markers, left axis) and for the difference between both polarizations

\section{APPENDIX A}

To find the condition for the two polarization responses to be uncorrelated we calculate the autocorrelation of the response (5) as a function of $\Delta \beta$ :

$$
\Gamma(t, \Delta \beta)=<R_{F U T}(t, \beta) R_{F U T}^{*}(t, \beta+\Delta \beta)>
$$

It is shown below that the magnitude of $\Gamma$ is independent of $t$. Hence, without loss of generality we substitute into (9) $t=0$ and the expression at the right hand side of (5):

$$
\begin{aligned}
\Gamma(0, \Delta \beta) \propto & \iint_{0}^{L}<r\left(z^{\prime}\right) r^{*}\left(z^{\prime \prime}\right)>e^{-j 2\left[\beta z^{\prime}-(\beta+\Delta \beta) z^{\prime \prime}\right]} \\
& R_{a}\left(\frac{2 z^{\prime}}{v_{g}}\right) R_{a}\left(\frac{2 z^{\prime \prime}}{v_{g}}\right) d z^{\prime} d z^{\prime \prime}
\end{aligned}
$$

recalling that $<r\left(z^{\prime}\right) r^{*}\left(z^{\prime \prime}\right)>\propto \delta\left(t^{\prime}-t^{\prime \prime}\right)$ we obtain:

$$
\Gamma(0, \Delta \beta) \propto \int_{0}^{L} e^{j 2 \Delta \beta z^{\prime}} R_{a}^{2}\left(\frac{2 z^{\prime}}{v_{g}}\right) d z^{\prime}
$$

It can be seen that $\Gamma$ is essentially a Fourier transform of $R_{a}^{2}\left(2 z^{\prime} / v_{g}\right)$. It is also apparent that in the general case where $t \neq 0$ the autocorrelation function $\Gamma(t, \Delta \beta)$ is modified by a phase factor only and retains its magnitude. Denoting the spatial width of $R_{a}^{2}\left(2 z^{\prime} / v_{g}\right)$ as $L_{a}$, two responses will be uncorrelated as long as their $\Delta \beta$ satisfies:

$$
\Delta \beta \gg \frac{\pi}{L_{a}}
$$

\section{ACKNOWLEDGMENT}

This work was supported in part by the Israeli ministry of science and technology and by Universidad Pública de Navarra, Feder funds and Spanish Ministerio de Economía y Competitividad through the project TEC2016-76021-1R 


\section{REFERENCES}

[1] A. Masoudi, M. Belal, and T. P. Newson, "A distributed optical fibre dynamic strain sensor based on phase-OTDR," Measurement Science and Technology, vol. 24, no. 8, p. 085204, 2013.

[2] D. Arbel and A. Eyal, "Dynamic optical frequency domain reflectometry," Opt. Express, vol. 22, no. 8, pp. 8823-8830, Apr 2014.

[3] W. Zou, S. Yang, X. Long, and J. Chen, "Optical pulse compression reflectometry: proposal and proof-of-concept experiment," Opt. Express, vol. 23, no. 1, pp. 512-522, Jan 2015.

[4] J. J. Mompó, S. Martín-López, M. González-Herráez, and A. Loayssa, "Sidelobe apodization in optical pulse compression reflectometry for fiber optic distributed acoustic sensing," Opt. Lett., vol. 43, no. 7, pp. 1499-1502, Apr 2018.

[5] M. Nazarathy, S. A. Newton, R. P. Giffard, D. S. Moberly, F. Sischka, W. R. Trutna, and S. Foster, "Real-time long range complementary correlation optical time domain reflectometer," Journal of Lightwave Technology, vol. 7, no. 1, pp. 24-38, Jan 1989.

[6] M. A. Soto, G. Bolognini, and F. D. Pasquale, "Long-range simplexcoded BOTDA sensor over $120 \mathrm{~km}$ distance employing optical preamplification,” Opt. Lett., vol. 36, no. 2, pp. 232-234, Jan 2011.

[7] M. A. Soto, G. Bolognini, F. D. Pasquale, and L. Thévenaz, "Simplexcoded BOTDA fiber sensor with $1 \mathrm{~m}$ spatial resolution over a $50 \mathrm{~km}$ range," Opt. Lett., vol. 35, no. 2, pp. 259-261, Jan 2010.

[8] D. Elooz, Y. Antman, N. Levanon, and A. Zadok, "High-resolution longreach distributed Brillouin sensing based on combined time-domain and correlation-domain analysis," Opt. Express, vol. 22, no. 6, pp. 64536463, Mar 2014.

[9] J. Mariñelarena, H. Iribas, and A. Loayssa, "Pulse coding linearization for Brillouin optical time-domain analysis sensors," Opt. Lett., vol. 43, no. 22, pp. 5607-5610, Nov 2018.

[10] D. Lee, H. Yoon, P. Kim, J. Park, N. Y. Kim, and N. Park, "SNR enhancement of OTDR using biorthogonal codes and generalized inverses," IEEE Photonics Technology Letters, vol. 17, pp. 163-165, 2005.

[11] D. Lee, H. Yoon, N. Y. Kim, H. Lee, and N. Park, "Analysis and experimental demonstration of simplex coding technique for SNR enhancement of OTDR," in Proceedings of the Lightwave Technologies in Instrumentation and Measurement Conference, 2004., Oct 2004, pp. $118-122$.

[12] L. Shiloh, N. Levanon, and A. Eyal, "Highly-sensitive distributed dynamic strain sensing via perfect periodic coherent codes," in 26th International Conference on Optical Fiber Sensors. Optical Society of America, 2018, p. TuE25.

[13] N. Arbel, L. Hirschbrand, S. Weiss, N. Levanon, and A. Zadok, "Continuously operating laser range finder based on incoherent pulse compression: Noise analysis and experiment," IEEE Photonics Journal, vol. 8, no. 2, pp. 1-11, April 2016.

[14] A. H. Hartog, "An introduction to distributed sensors and static measurement," in Distributed vibration sensors and static measurement. CRC Press, 2017, pp. 257-261

[15] M. Ren, P. Lu, L. Chen, and X. Bao, "Theoretical and experimental analysis of $\phi$-OTDR based on polarization diversity detection," IEEE Photonics Technology Letters, vol. 28, no. 6, pp. 697-700, 2016.

[16] N. Levanon and E. Mozeson, "Radar Signals." WILEYINTERSCIENCE, 2004, pp. 53-73.

[17] S. W. Golomb, "Two-valued sequences with perfect periodic autocorrelation," IEEE Transactions on Aerospace and Electronic Systems, vol. 28, no. 2, pp. 383-386, April 1992.

[18] Z. Wang, L. Zhang, S. Wang, N. Xue, F. Peng, M. Fan, W. Sun, X. Qian, J. Rao, and Y. Rao, "Coherent $\phi$-OTDR based on I/Q demodulation and homodyne detection." Optics express, vol. 24, no. 2, pp. 853-8, jan 2016.

[19] D. Shahar, "Minimizing the variance of a weighted average," Open Journal of Statistics, vol. 7, pp. 216-224, 2017. 